\title{
On the Interference Immunity of Visible Light Communication (VLC)
}

\author{
Antonio Costanzo \\ Inria Lille - Nord Europe \\ antonio.costanzo@inria.fr
}

\author{
Valeria Loscrí \\ Inria Lille - Nord Europe \\ valeria.loscri@inria.fr
}

\author{
Virginie Deniau \\ Université Gustave Eiffel \\ virginie.deniau@univ-eiffel.fr
}

\author{
Jean Rioult \\ Université Gustave Eiffel \\ jean.rioult@univ-eiffel.fr
}

\begin{abstract}
Visible Light Communication (VLC) relies on the dual use of existing light infrastructure for wirelessly send data. VLC is regarded as a "green", cost-effective and secure communication technology. Moreover, VLC has been always considered immune to Radio Frequency (RF) waves. The only type of interference that has always been included in the channel model is the optical interference. Nevertheless, all the interference components potentially impacting on the performance of the system should be integrated in the transmitting model in order to improve the performance of the communication system. In this paper, we investigate on the interference and immunity of the VLC systems in respect of frequencies spanning the spectrum up to $1 \mathrm{GHz}$. We provide a detailed evaluation of the immunity of a VLC system by the means of experiments realized in "controlled" environment, i.e. an anechoic chamber, by considering interference at different power levels. Results are very interesting, since at specific frequency values, the VL transmitted signal results too "interfered" and noisy to be correctly recovered. In order to evaluate the impact of this interference, we have estimated the Bit Error Rate (BER) for different index modulations of PulsePosition Modulation (mPPM).
\end{abstract}

Index Terms - Visible Light Communication, Electromagnetic Interference, Pulse-Position Modulation

\section{INTRODUCTION}

The dual use of light for illumination and for wireless information has recently garnered attention from the wireless networks community. Visible Light Communication (VLC) is recognized as a green communication technology solution and as a safe technology to human people in comparison of Radio Frequency (RF) based technology. The growing research interest in optical wireless spectrum relies on the need to find a complementary technology to the conventional one based on $\mathrm{RF}$ in order to overcome the spectrum scarcity and to meet the high data rates required. Among the positive characteristics associated to VLC, are a wider bandwidth, an inherent security and no RF electromagnetic frequency interference. It is wellknown that VLC is vulnerable to the optical interference noise. A previous analysis with characterization and modeling of artificial light interference on optical wireless communication system has been proposed in [1]. In particular, the authors present a characterization based on extensive measurements of the artificial light interference on wireless indoor infrared transmission systems. The authors prove the importance of including this interference in the optical wireless indoor channel model in order to enhance the performance of the communication system above all for systems operating at high rate of several Mbit/s. In [4] the authors propose a signal processing mitigation approach for "mitigating" the impact of external light sources and improving the performance of the VLC in terms of Bit Error Rate (BER). The authors of [5] consider the external interference and propose a learning approach integrated in the VLC system in order to improve its robustness. In particular, the optimal preamble length of the synchronization message is computed by modeling the correct length selection as a multi-arm bandit. In [2] authors consider the issue of overlapping light sources and how is possible to recover the signals. In particular, they propose a physical protocol layer for identifying interference regions and isolate them from the transmission. Inter-Symbol Interference (ISI) in VLC is considered in [3], where the authors focus on Multiple-Input-Multiple-Output (MIMO) approaches in order to increase the channel capacity. Specifically, they analyze the ISI caused by the physical proximity of the photodetectors (PDs) by evaluating the impact of different parameters. In [7] the authors consider a pair of LEDs interfering each other and rely on a simple nanoslit metasurface to increase the directivity of the transmitting beams in order to reduce the interference effect. Authors in [6] propose an approach based on angular diversity receivers in order to mitigate the interference in optical attocel networks. In [8] the authors focus on the specific and unique features of optical channel in VLC and their interference. The authors consider multi access points systems that create additional interference.

So far, the main contributions on VLC interference focus on noise and interference deriving from other natural/artificial light sources. A different perspective of interference is introduced in [9]-[11], where the authors consider the effect of ripple interference on the receiver filter. They highlight as ripple is a source of harmful interference for VLC systems and propose a new BER expression to account for this aspect. Moreover, a low-pass filter is proposed to alleviate the ripple interference effect on VLC systems.

In this paper, we focus on the immunity to electromagnetic interference. In particular, we consider a VLC system constituted by a transmitter (LED) and a receiver (photodiode), and we analyze the system from an interference point of view. In practice, we consider a completely controlled suite of experiments in an anechoic chamber, with and without external interference as an antenna transmitting a signal at different frequencies up to $1 \mathrm{GHz}$ and at different powers. Results are 
surprising, since at certain frequencies, we detect very high interference. In order to evaluate the impact of the interference from a communication point of view, we have implemented different modulation schemes, m-Pulse Position Modulation ( $\mathrm{mPPM}$, with $\mathrm{m}=2,4,8,16$ ) and we have considered different scenarios to identify the specific conditions impacting on the communication system. To the best of our knowledge, this is the first paper focusing on RF electromagnetic immunity of VLC. The rest of the paper is organized as follows. In Section II we present our prototype and the main parameters of the experiments. Section III gives the preliminary results motivating the work. In Section IV we show the experiments realized in the anechoic chamber by spanning on different frequencies in the spectrum. In $\mathrm{V}$ we evaluate the impact of the interference on the Bit Error Rate (BER) by considering different power levels. Finally, we conclude the paper in SectionVI.

\section{System SetuP}

In this section we detail the components of our communication system based on Visible Light, the modulations techniques implemented and the specific environment where the measurements have been realized. The VLC system is characterised with a LED as transmitter and a photodiode as receiver. A specific circuit has been designed and implemented to drive the LED lamp in the transmitting stage and another one for conditioning the signal delivered to the photodiode. A Software Defined approach has been used for modulating, demodulating and processing the signal. Two Universal Software Radio Peripheral (USRP 2922), integrated with two low frequency daughterboards (LFTX and LFRX) have been used. All the operations (e.g. modulation, demodulation, filtering) are implemented using the commercial software Labview. The experiments have been performed in an anechoic chamber with a setup as represented in Figure 1. In all the experiments, we applied a fixed distance of 1 meter between the transmitter and the receiver. Both USRPs have been kept outside the anechoic chamber and only the communication system was inside the chamber. In the anechoic chamber, two types of experimentation were carried out. In a first step, we activated the VLC system and measured the emissions of the system with a wide frequency band antenna and a spectrum analyzer. Cable interconnections, lead-bond wires, power distribution networks and other sources of radiated emissions could potentially affect the system. In this test we quantified these effects in terms of amplitude and frequency. Experimentation have been carried out up to $5 \mathrm{GHz}$, considering three different modulations. Since no significant effects have been observed in the the range $[1-5] \mathrm{GHz}$, results are presented in Figure 3 up to $1 \mathrm{GHz}$. The most important radiated emissions of the VLC system are at 222, 444 and $666 \mathrm{MHz}$. These are signals that are produced by the VLC system during its operation and are radiated by the components or printed tracks in which the signals travel. These frequencies can therefore be considered as "useful" frequencies for the system. In a second step, the wide band antenna was connected to a generator to emit radio- frequency interference in the anechoic chamber. The power of the radio frequency interference was controlled and adjusted by using a triaxial Electric field probe located near by the VLC system. In these tests, we were particularly interested in radio frequency interference covering the frequencies of 222 , 444 and $666 \mathrm{MHz}$.

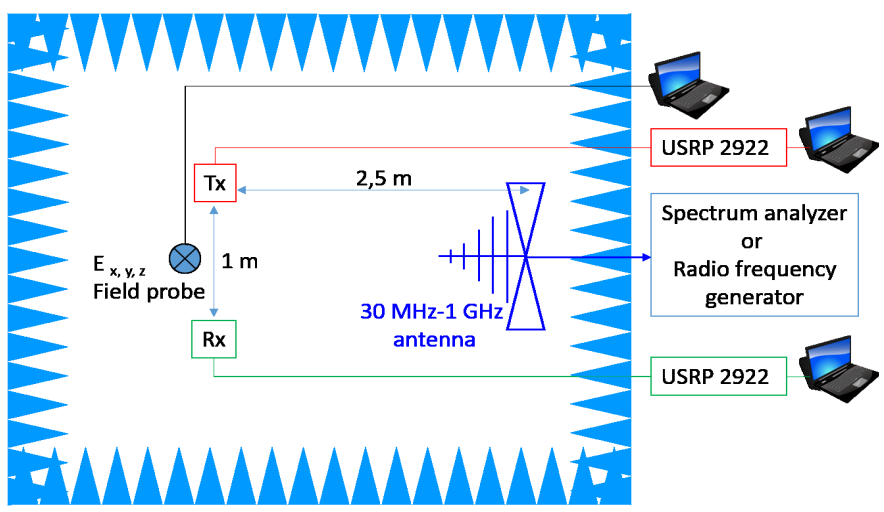

Fig. 1. Setup of the VLC system in the anechoic chamber.

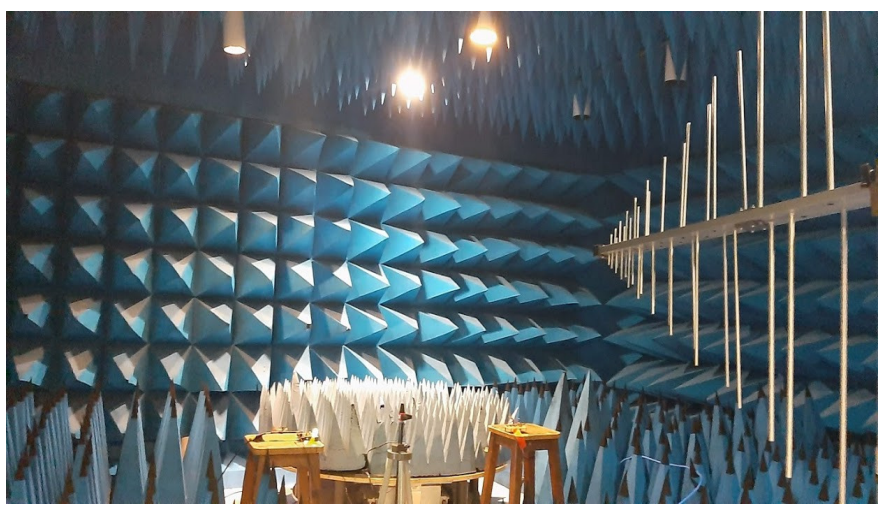

Fig. 2. Tests on the effects of the interference in a VLC system in anechoic chamber

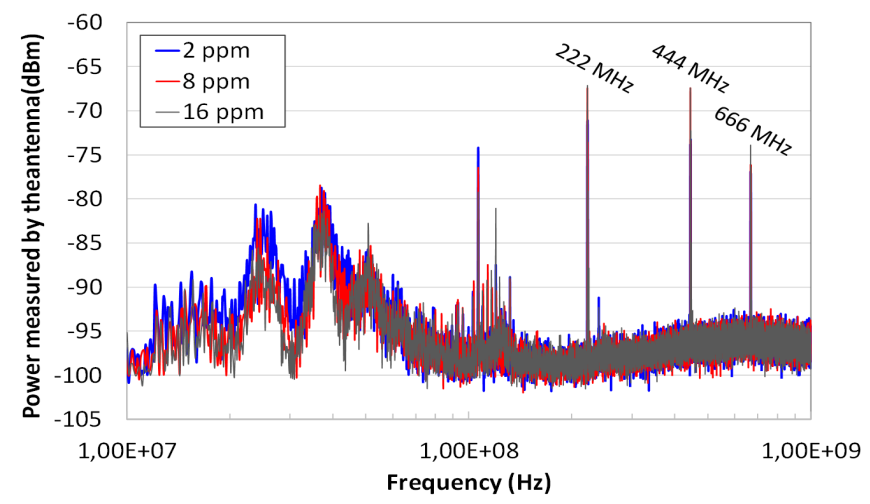

Fig. 3. Spectrum of electromagnetic emission generated by the VLC system

The main optical and electrical parameters of VLC receiver and transmitter are summarized in Table. I. 
TABLE I

EXPERIMENTAL VALIDATIONS: MAIN PARAMETERS OF TRANSMITTING AND RECEIVING HARDWARE STAGES

\begin{tabular}{|c|c|c|c|}
\hline \multicolumn{2}{|c|}{ Receiver } & \multicolumn{2}{|c|}{ Transmitter } \\
\hline $\begin{array}{l}\text { Photodiode } \\
\text { Model }\end{array}$ & $\begin{array}{c}\text { CENTRONIC } \\
\text { OSD15-5T }\end{array}$ & $\begin{array}{l}\text { LED } \\
\text { Model }\end{array}$ & $\begin{array}{c}\text { CREE } \\
\text { MKRAWT } \\
-02-0000 \\
-0 \text { D00J2051 }\end{array}$ \\
\hline Active Area & $15 \mathrm{~mm}^{2}$ & Maximum Power & $15 \mathrm{~W}$ \\
\hline $\begin{array}{l}\text { Responsivity } \\
(436 \mathrm{~nm})\end{array}$ & $0.18-0.21$ & $\begin{array}{c}\text { Effective } \\
\text { Power }\end{array}$ & $4 \mathrm{~W}$ \\
\hline Dark Current & $1-5 \mathrm{nA}$ & $\begin{array}{l}\text { Maximum } \\
\text { Light Flux }\end{array}$ & $1040 \mathrm{~lm}$ \\
\hline Field of View & $45 \mathrm{deg}$ & Field of View & 120deg \\
\hline $\begin{array}{c}\text { Noise } \\
\text { Equivalent } \\
\text { Power } \\
(436 \mathrm{~nm})\end{array}$ & $5.5 * 10^{-14}$ & Forward Voltage & $12 \mathrm{~V}$ \\
\hline Capacitance & $80 \mathrm{pF}$ & $\begin{array}{c}\text { Maximum } \\
\text { Forward Current }\end{array}$ & $1200 \mathrm{~mA}$ \\
\hline Bandwidth & $29.1 \mathrm{MHz}$ & $\begin{array}{c}\text { Maximum } \\
\text { Switching Speed }\end{array}$ & $35 \mathrm{MHz}$ \\
\hline $\begin{array}{c}\text { Shunt } \\
\text { Resistance }\end{array}$ & $200 M \Omega$ & Reverse Voltage & $5 \mathrm{~V}$ \\
\hline Rise Time & $12 \mathrm{~ns}$ & Number of LED & 5 \\
\hline $\begin{array}{c}\text { Trans } \\
\text { Impedance } \\
\text { Amplifier Gain }\end{array}$ & $1 \mathrm{M} \Omega$ & $\begin{array}{c}\text { Optical } \\
\text { Concentrator } \\
\text { Gain }(6 \mathrm{~m})\end{array}$ & 5 \\
\hline
\end{tabular}

\section{Preliminary Experimental Results}

In this section we present the preliminary results we have realized with our VLC system and an interfering antenna that motivate us to deeply evaluate the effects of the interference on the VLC system. The interference effects have been evaluated both in the time and frequency domain and the impact has been measured in terms of Bit Error Rate (BER), considering an active communication between the transmitter and the receiver based on Pulse Position Modulation (PPM) with different index $\mathrm{m}$. In the first step of experimental tests, the effects of a $10 \mathrm{dBm}$ interfering signal, generated at different frequencies, without active communication between transmitter and receiver have been measured. We assumed $T_{o b s}=1 \mathrm{~s}$ as observation time and $S=1 \mathrm{M}$ sample/s as sample rate.

The received signal is shown, in time domain, in Fig. 4.

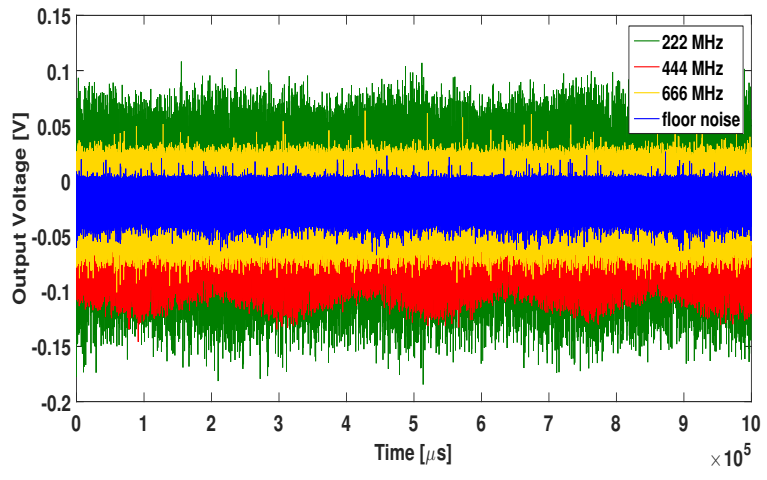

Fig. 4. Effects of $10 \mathrm{dBm}$ interference signal on the proposed VLC system without active communication between transmitter and receiver: Time Domain
A floor noise, mainly due to shot noise and thermal noise, affects the system, even if no transmission and no other interfering signal (optical or electrical) are active in the scenario. Since shot noise also depends by the light level in the environment (included the transmitting light itself), a first series of measurement have been produced by considering frequencies test at step of $5 \mathrm{MHz}$, without active communication and in a "dark" environment. In this way, we are able to verify if interfering radiated signals couple the devices and produce some negative effects on the system performance. Most of the frequencies we considered do not produce a significant effect: in this case, the overall detected signal is only due to floor noise. However, for certain frequency values shown in Fig. 4, namely $222 \mathrm{MHz}, 444 \mathrm{MHz}$ and $666 \mathrm{MHz}$ the overall level of interference significantly overpasses the level of floor noise, even if a weak level of radiating power $(10 \mathrm{dBm})$ is used. In order to understand the spectral content of these interference, power spectral density of the received signal has been calculated and shown in Fig.5 .

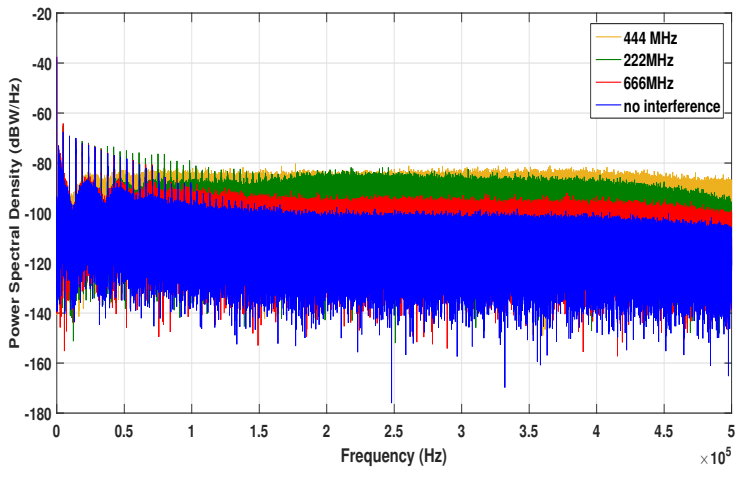

Fig. 5. Effects of $10 \mathrm{dBm}$ interference signal on the proposed VLC system without active communication between transmitter and receiver: Frequency Domain

Since floor noise is mainly characterized as a Gaussian distribution, its spectrum covers all the operating frequency range of our Visible Light Communication system. However, also the effect of interference is distributed in the spectrum at the same way. So, a radiated source at $222 \mathrm{MHz}, 444 \mathrm{MHz}$ and $666 \mathrm{MHz}$, can severely impact on the communication between VLC transmitter and receiver. Using the same amplitudes and the same frequencies of interfering signals of previous tests, a 16PPM communication has been activated between the transmitter and the receiver. The width of each pulse has been chosen equal to $\tau=5$ samples $=5 \mu \mathrm{s}$. In order to understand how communication is affected by interference, we have considered an observation time equal to to $200 \mu \mathrm{s}$ and the received pulses are compared in Fig. 6 in time domain. In this case, is evident how interference modifies the correct shape of receiving signal. The power spectral density, associated to each received signal, is shown in Fig. 7. We can remark important changes in spectral components if the signal is affected by interference. In particular, an increment of lateral lobes level affects the normal cardinal sine evolution associated to the 


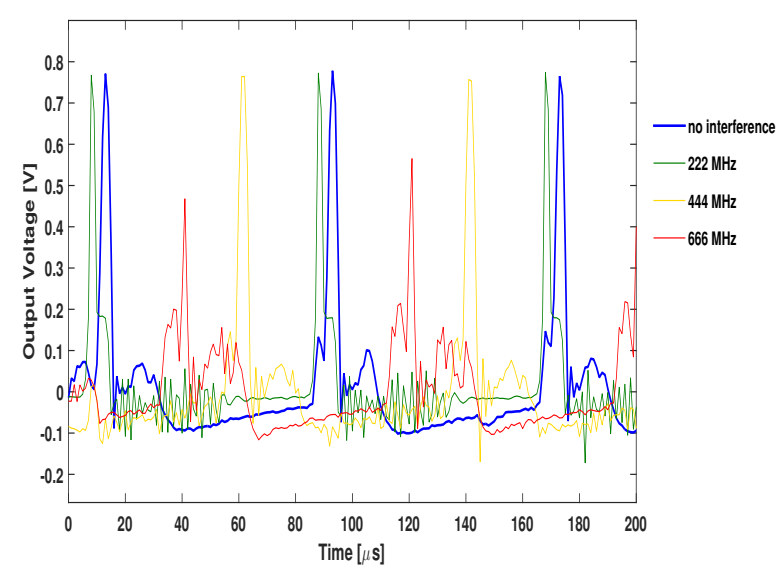

Fig. 6. Effects of $10 \mathrm{dBm}$ interference signal on the proposed VLC system with 16 PPM active communication between transmitter and receiver: Time Domain

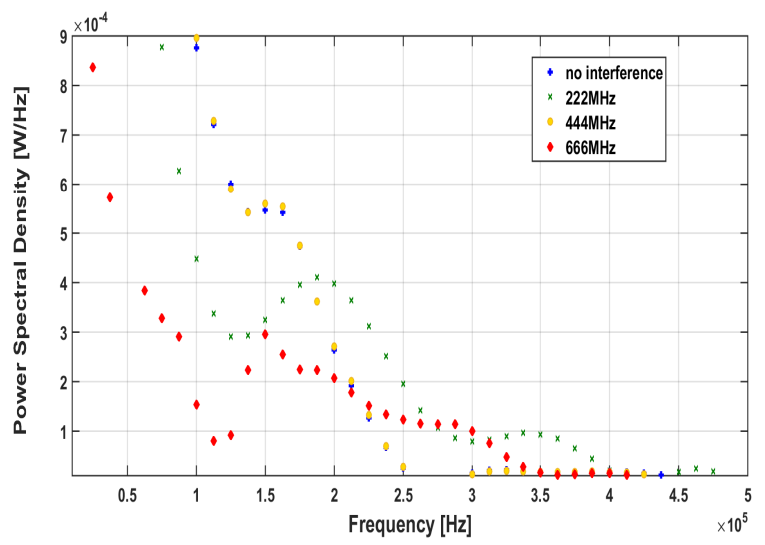

Fig. 7. Effects of $10 \mathrm{dBm}$ interference signal on the proposed VLC system with 16 PPM active communication between transmitter and receiver: Frequency Domain

pulsed signal.

\section{INTERFERENCE EFFECTS ON BIT ERROR RATE: FREQUENCY TUNING}

In order to better evaluate the effects of the interfering signal, we have performed a suite of targeted tests in the anechoic chamber, by generating an electrical field equal to $E_{0}=5 \mathrm{~V} / \mathrm{m}$ and varying the frequencies in a wider range. We tested the effects of these radiating interference of the communication performance on 2, 4, 8 and 16 PPM. In particular, we estimate the Bit Error Rate performed by the communication system on the received data. Since several campaign of experimental tests have been provided, a limited amount of data (equal to 1Msamples) has been collected for each test. However, in order to measure a bit error rate with high precision, a very long observation should be set, because the minimum BER value is inversely proportional to the number of received symbols. For that reason, we estimate the BER, starting by received signal, instead of directly measuring it. The first step is the estimation of Signal to Noise plus Interference Ratio (SINR), which is defined as the power of a signal of interest divided by the sum of the interference power (from all the other interfering signals) and the power of the noise affecting the system. In our case, we consider three main contributions:

- The power associated to the signal of interest, $S$, is the power of PPM modulated signal received by the optical front-end, which allows the reconstruction of useful information.

- The power associated to the overall noise, $N$ is constituted by the shot noise (generated by the LED itself) and the thermal noise (generated by the amplifiers and the other components in the receiver) [13].

- The interference, $I$, due to the effects caused by radiating electric fields (i.e. an antenna emitting a radiation) we introduced in the environment. No optical interference are considered in this analysis.

So, we can express SINR as:

$$
S I N R=\frac{S}{N+I}
$$

It is worth to precise that we have considered different polarization for the antenna, but we have not remarked differences in terms of interference on the VLC system and for that, the results presented in this paper are based on vertical polarization of the radiating antenna. For having an immediate synchronization and a comfortable analysis, in all the modulation schemes we used, we transmitted repeatedly the first symbol of the related alphabet. In particular, when 2PPM modulation is tested, a repetition of the symbol 0 is transmitted; in the same way, for the 4PPM the symbol 00, for 8 PPM the symbol 000 and for the 16PPM the symbol 0000 are transmitted. In this case, the useful signal is composed by a train of peaks, each peak delayed of a quantity $\mathrm{T}$, equal to the symbol time. Being $\mathrm{M}$ the order of modulation and $\tau$ the duration of each pulse set to $5 \mu s$ for each modulation, symbol time can be expressed as:

$$
T=M \tau
$$

In order to obtain an estimation of the power associated to the useful signal in the whole transmitting time, we integrate the received $x(t)$ signal in the interval where the signalling pulse is supposed to be, and we consider an average between the power associated to all the received pulses. Being $\mathrm{P}$ the total number of transmitted symbols (and transmitted pulses), and $\mathrm{p}$ the index of each symbol, we estimate the power associated to the signal as:

$$
S=\frac{\sum_{p=0}^{P-1} \frac{1}{\tau} \int_{p T}^{p T+\tau}|x(t)|^{2} d t}{P}
$$

All the power associated to the received signal, which falls outside one of the time slots $[p T, p T+\tau], \tau \in[0, P-1]$, 
contributes to increase the term N+I. The estimation of the average power associated to noise and interference, becomes:

$$
N+I=\frac{\sum_{p=0}^{P-1} \frac{1}{\tau} \int_{p T+\tau}^{(p+1) T}|x(t)|^{2} d t}{P}
$$

Even if both thermal and shot noise, compounding the term $\mathrm{N}$, follow a Gaussian behavior [13], the analytic description of interference I in optical systems is much more complicated, and a Gaussian assumption can lead to significant error of the analysis [12]. However, in this specific work, tests are produced into an anechoic chamber with no external lighting devices in the environment. Since external optical interference is negligible, the only significant interference is the one produced by RF test emissions on the transmitting and receiving circuits, which seems to affect the received signal into respect optical interference in the same way as thermal noise does. This effect is evidently shown in preliminary results ( Fig. 4 and Fig 5)). Since multiple AWGN components (shot noise, thermal noise and RF emission effects) can be observed, we can consider total noise variance as the summation of individual noise variances due to properties of the Gaussian distribution [12]. According to this assumption, we can process the estimation of the effects of noise and interference on Bit Error Rate in the same way if we treat a Gaussian noise. The overall Bit Error Rate is calculated, starting by SINR, as follows:

$$
B E R=\frac{1}{2} \operatorname{erfc}\left(\frac{1}{\sqrt{2}} \sqrt{\left.\operatorname{SINR} \frac{M}{2} \log _{2} M\right)}\right.
$$

being $\operatorname{erfc}$ the operator corresponding to the Complementary Error Function, namely:

$$
\operatorname{erfc}(x)=\int_{0}^{x} e^{-t^{2}} d t
$$

Even if this technique represents an approximation of the exact Bit Error Rate, it is really useful for having a fast estimation, suitable for real time operations. Indeed, the quantity of data needed for obtaining BER in this way is much lower than the one necessary for a direct counting of incorrect symbols. In Fig. 8 we show the BER associated to each order of modulation, adding, for each measurement, an interfering signal with a progressive frequency in the range $[50 \mathrm{MHz}$ $1 \mathrm{GHz}]$.

It is evident how interfering signals with different frequencies cause different effects on system performance. Worst performance have been obtained at $222 \mathrm{MHz}, 444 \mathrm{MHz}$ and 666 $\mathrm{MHz}$. Since the two higher frequencies are centered in integer multiple values, into respect to the lowest one, it is reasonable to consider these effects as generated by the same interfering phenomenon, which produces three different harmonics with a significant power level.

\section{INTERFERENCE EFFECTS ON BIT ERROR RATE WITH DIFFERENT LEVELS OF POWER}

In this section we evaluate the effect of the power variation of the interference on the communication system, by considering the same setup as in Fig. 2. We compare a case when

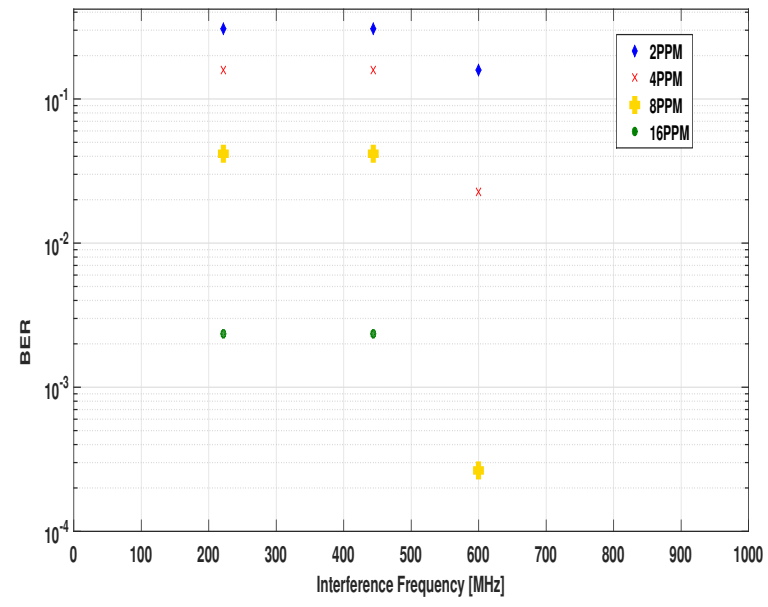

Fig. 8. Effects on BER produced by $5 \mathrm{~V} / \mathrm{m}$ interference signal on the proposed VLC system, considering 2,4,8 and 16 PPM modulation: zoom on most critical frequencies

interference does not affect the performance of the system (frequency of interference equal to $250 \mathrm{MHz}$ ), and a critical case just a bit further $(222 \mathrm{MHz})$. The power of electric field has been tuned, for each experiment, in the range $[0-10 \mathrm{~V} / \mathrm{m}]$, considering a step of $0.1 \mathrm{~V} / \mathrm{m}$ in the range $0-1 \mathrm{~V} / \mathrm{m}$ and a step of $1 \mathrm{~V} / \mathrm{m}$ in the range $1-10 \mathrm{~V} / \mathrm{m}$. Results in time domain are shown, respectively, in Fig. 9 and 10. For a clear representation of the time evolution of the pulses, signal received using a 8PPM modulation have been considered.

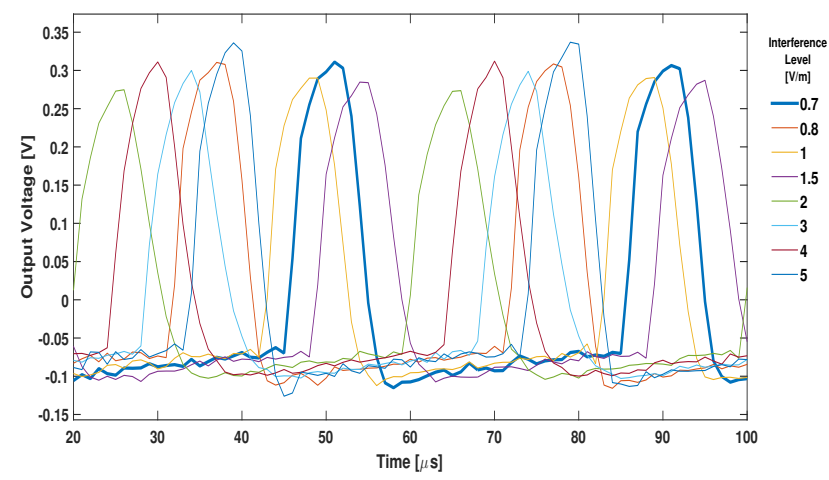

Fig. 9. Effects on pulse shape produced by $250 \mathrm{MHz}$ interference signal on the proposed VLC system, considering 8 PPM modulation

We observe how, in the first case (Fig. 9), no effects can be appreciated even if the level of interfering reaches its maximum value. However, in the second series of measurements (Fig. 10), a devastating effect on received signal is already obtained for low values of emissions. When the external electrical field reaches a level of $2 \mathrm{~V} / \mathrm{m}$, received signal is completely flooded by interference. For this motivation, we stopped the analysis, in this case, to the above mentioned level of interfering field intensity. In this latter case, Bit Error Rate has been shown for each value of field amplitude, considering 


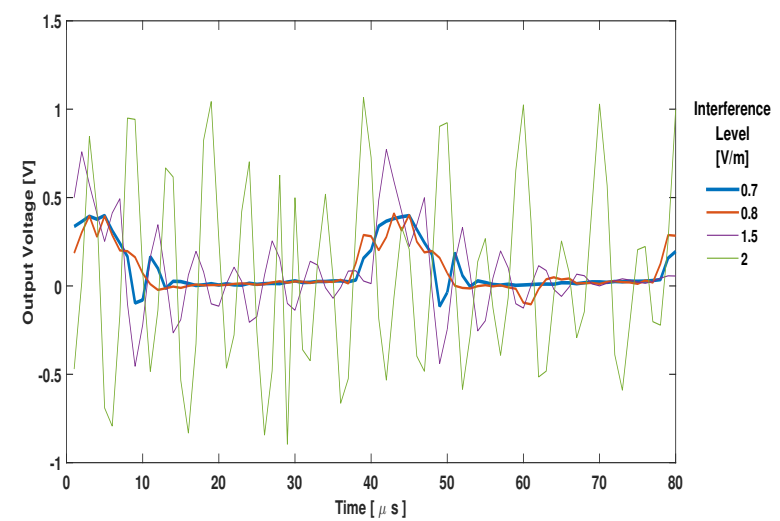

Fig. 10. Effects on pulse shape produced by $222 \mathrm{MHz}$ interference signal on the proposed VLC system, considering 8PPM modulation

\section{2, 4, 8 and 16 PPM.}

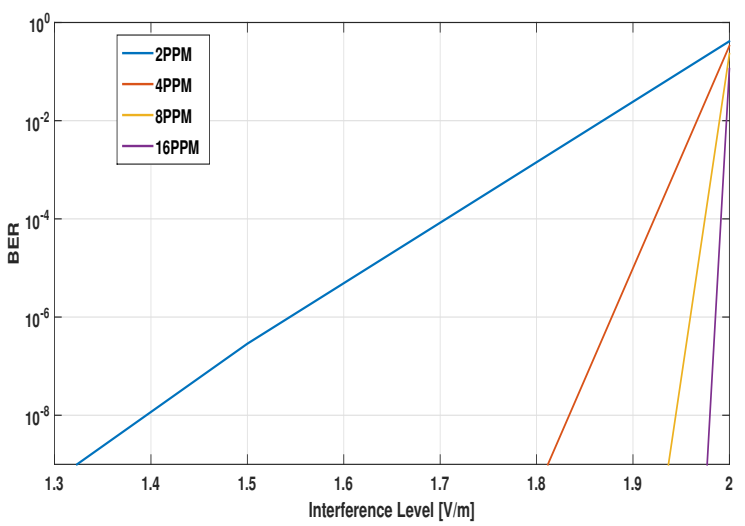

Fig. 11. Effects on BER produced by $222 \mathrm{MHz}$ interference signal on the proposed VLC system, considering 2,4,8 and 16 PPM modulation

In this case, we observe a significant effect on system performance, which is directly related to the amplitude of interfering field, with a complete lost of information in the worst cases since the BER is too high to correctly decode the received data. Based on these results, we argue as the integration of this type of effects in the channel model is of paramount importance ion order to experience undesired degradation in the VLC system.

\section{CONCLUSIONS}

In this paper we have implemented a VLC prototype with a Software Defined approach in order to evaluate the interference immunity of the communication system. In particular, we have implemented different index of PPM technique and tested the system in an anechoic chamber by spanning on different frequency values and different power levels. Results obtained are surprising, since the VLC system seems very sensible to specific frequencies. In order to evaluate the impact of the interference, we have evaluated the Bit Error Rate (BER) and we have shown that for certain "critical" frequencies, the BER is too high to be able to correctly recover the transmitted data. These results are important in order to include this type of interference in the channel model and improve link performance through new signal process approaches. Indeed, as future works, we plan to better characterise this type of interference with more extensive measurements with higher power levels and a wider frequency range, extending the analysis to other modulation techniques, like Orthogonal Frequency-Division Multiplexing (OFDM). Moreover, we will design some ad hoc signal processing technique in order to mitigate the effect of this type of interference.

\section{ACKNOWLEDGMENT}

This work was supported primarily by the Région Hauts de France under "STIMuLE: Support for Interdisciplinary, Multi-establishment and Exploratory Research" Agreement No. 19004007.

\section{REFERENCES}

[1] A. J. C. Moreira, R. T. Valadas and A. M. de Oliveira Duarte "Characterisation and modelling of artificial light interference in optical wireless communication systems," Proceedings of 6th International Symposium on Personal, Indoor and Mobile Radio Communications, Toronto, Ontario, Canada, 1995, pp. 326-331 vol.1.

[2] Guler, Ali Ugur, Tristan Braud and Pan Hui. "Spatial Interference Detection for Mobile Visible Light Communication.” 2018 IEEE International Conference on Pervasive Computing and Communications (PerCom) (2017): 1-10.

[3] Jingyuan Feng, Chunyong Yang, Jin Hou, Hao Long, Shaoping Chen, Performance enhancement for indoor visible light communication system with an improved inter-symbol interference model using optimized hemispherical optical-angle-diversity-receivers, Optics Communications, Volume 454, 2020.

[4] Costanzo, Antonio, Valeria Loscri, and Mauro Biagi. "A Noise Mitigation Approach for VLC Systems.” 2019 Global LIFI Congress (GLC). IEEE, 2019.

[5] A. Costanzo and V. Loscri, "A Learning Approach for Robust Carrier Recovery in Heavily Noisy Visible Light Communication," 2019 IEEE Wireless Communications and Networking Conference (WCNC), Marrakesh, Morocco, 2019, pp. 1-6.

[6] Chen, Z, Basnayaka, D, Wu, X and Haas, H., 'Interference Mitigation for Indoor Optical Attocell Networks using Angle Diversity Receiver, Journal of Lightwave Technology, vol. 36, no. 18, 2018. https://doi.org/10.1109/JLT.2018.2848221

[7] Constantinos Valagiannopoulos, Theodoros A Tsiftsis and Vassilios Kovanis, "Metasurface-enabled interference mitigation in visible light communication architectures," in Journal of Optics, Volume 21, Number 11, Oct. 2019.

[8] Abdalla I, Rahaim MB, Little TDC, "Interference in multi-user optical wireless communications systems. Phil. Trans. R. Soc. A 378: 20190190, 2020. http://dx.doi.org/10.1098/rsta.2019.0190

[9] X. Deng, Y. Wu, K. Arulandu, G. Zhou and J. P. M. G. Linnartz, ’Performance comparison for illumination and visible light communication system using buck converters," 2014 IEEE Globecom, Austin, TX, 2014, pp. 547-552.

[10] X. Deng, J. P. M. G. Linnartz, K. Arulandu, G. Zhou and Y. Wu, "Effect of buck driver ripple on BER performance in visible light communication using LED,' 2015 IEEE International Conference on Communication (ICC), London, 2015, pp. 1368-1373.

[11] X. Deng, K. Arulandu, Y. Wu, G. Zhou and J. P. M. G. Linnartz, ”System modeling and analysis for LED-based Visible Light Communication using Buck Driver,' IEEE Transactions on Communications, vol. PP, no. 99, pp. 1-1, 2018.

[12] M. Rahaim and T. D. C. Little, "Optical interference analysis in Visible Light Communication networks," 2015 IEEE International Conference on Communication Workshop (ICCW), London, 2015, pp. 1410-1415.

[13] S. Dimitrov, H.Haas, "Principles of LED Light Communications: Towards Networked Li-Fi", Cambridge University Press, 2015 